\title{
ON THE DISTRIBUTION OF THE MAXIMAL CLIQUE SIZE FOR THE VERTICES IN REAL-WORLD NETWORK GRAPHS AND CORRELATION STUDIES
}

\author{
Natarajan Meghanathan \\ Jackson State University, 1400 Lynch St, Jackson, MS, USA \\ natarajan.meghanathanejsums.edu
}

\begin{abstract}
The high-level contributions of this paper are as follows: We modify an existing branch-andbound based exact algorithm (for maximum clique size of an entire graph) to determine the maximal clique size that the individual vertices in the graph are part of. We then run this algorithm on six real-world network graphs (ranging from random networks to scale-free networks) and analyze the distribution of the maximal clique size of the vertices in these graphs. We observe five of the six real-world network graphs to exhibit a Poisson-style distribution for the maximal clique size of the vertices. We analyze the correlation between the maximal clique size and the clustering coefficient of the vertices, and find these two metrics to be poorly correlated for the real-world network graphs. Finally, we analyze the Assortativity index of the vertices of the real-world network graphs and observe the graphs to exhibit positive assortativity with respect to maximal clique size and negative assortativity with respect to node degree; nevertheless, we observe the Assortativity index of the real-world network graphs with respect to both the maximal clique size and node degree to increase with decrease in the spectral radius ratio for node degree, indicating a positive correlation between the maximal clique size and node degree.
\end{abstract}

\section{KEYWORDS}

Maximal Clique Size, Node Degree, Correlation, Assortativity Index, Distribution, Network Graphs, Clustering Coefficient

\section{INTRODUCTION}

Network Science is an emerging area of research interest to study complex real-world networks from a graph theoretic point of view. We abstract the complex network as a graph with the nodes representing the vertices and the connections between any two nodes in the network modeled as edges in the graph. It is imperative that the algorithms run on these large scale graphs be as efficient as possible and do not take significant time to determine the metrics of interest. Though there exists efficient polynomial-time algorithms to determine widely studied metrics [1] like centrality, diameter, clustering coefficient, etc on these graphs, there still exists certain metrics like clique such that the problem of determining a maximum size clique is NP-hard [2]. A clique on a graph is a subset of the vertices such that there exists an edge between any two vertices in this subset; an algorithm to find cliques of various sizes could be used to identify closely-knit communities [3-5] of various sizes (constituent nodes) in real-world network graphs. 
The "maximum size clique" for a graph of $n$ vertices is a clique of the largest size $k(k \leq n)$ such that there does not exist a clique of size $k+1$ in the graph. A "maximal size clique for a vertex $i$ " in a graph is the clique of the largest size that involves vertex $i$ as one of the constituent vertices. While the maximum size clique for a graph is the maximal size clique for its constituent vertices, there could exist several other vertices in the graph for which the maximal size clique is smaller than the maximum size clique. Most of the research focus in the literature is to develop exact algorithms that could determine the maximum size clique for the entire graph as efficiently as possible with respect to both time and space complexity. Very little attention has been made to determine the maximal size cliques for the individual vertices in the graph. Specifically, to the best of our knowledge, no attempt has been made to analyze the distribution of the maximal clique sizes of the individual vertices in the real-world complex network graphs. In this paper, we choose a recently proposed exact algorithm [6] to determine the size of the maximum clique for large-scale complex network graphs and extend it to determine the size of the maximal clique that a particular node is part of. Using the exact algorithm to determine maximal clique size for the individual vertices of the graphs, we determine the distribution of the maximal clique size for all the six real-world network graphs considered in this study. We observe that five of the six realworld network graphs (irrespective of their number of nodes and degree distribution) exhibit a Poisson-style distribution for the maximal clique size; this is a significant observation that has not been hitherto reported in the literature.

The second half of our paper focuses on identifying a computationally-light metric for the individual nodes of a graph that correlates well (either positively or negatively) to that of the maximal clique size (which we categorize as a computationally-hard metric, owing to the NPhard nature of the problem to determine this metric and the significant time complexity involved in the exact algorithms for this metric). Once we identify such a computationally-light metric that correlates well with the maximal clique size of the vertices in complex network graphs, we could infer a ranking of the vertices based on this computationally-light metric as a ranking of the vertices based on the maximal clique size. To the best of our knowledge, we have not come across any such study to identify a computationally-light metric that correlates well with the maximal clique size for real-world network graphs. Ours is the first attempt in this direction. The two candidate computationally-light metrics that we consider are the clustering coefficient and the node degree. The clustering coefficient of a vertex is the ratio of the number of edges between the neighbors of the vertex to that of the maximum number of edges possible between the neighbors of the vertex. Our conjecture is that nodes that are part of a larger clique are more likely to have a larger clustering coefficient and vice-versa. Similarly, we conjecture that nodes that have a larger degree (number of neighbors) are likely to be part of cliques of larger size and vice-versa. Results of our correlation studies on real-world network graphs reveal that the maximal clique size has good correlation with node degree (especially as the variation in the node degree increases), whereas the maximal clique size correlates poorly with the clustering coefficient. We further confirm the positive correlation between the maximal clique size and node degree through an analysis of the Assortativity index of the vertices [1] in the real-world network graphs with respect to these two metrics. We observe the real-world network graphs could be ranked in a similar order in the decreasing order of the Assortativity index of the vertices with respect to both the maximal clique size and the node degree.

The rest of the paper is organized as follows: Section 2 describes related work on analysis of complex network graphs using cliques. Section 3 describes an efficient exact algorithm to determine the maximum clique size for an entire graph and our extension to determine the maximal clique size for the individual vertices of the graph. Section 4 presents the real-world network graphs studied in this paper and an analysis of their degree distribution and distribution of the maximal clique size of the vertices. Section 5 presents the results of the correlation studies between the maximal clique size and clustering coefficient. Section 6 presents the results of the 
correlation studies between the maximal clique size and the node degree. Section 7 presents the results of Assortativity index-based analysis of the real-world network graphs with respect to maximal clique size and node degree. Section 8 concludes the paper. Throughout the paper, we use the terms 'node' and 'vertex', 'link' and 'edge' interchangeably. They mean the same.

\section{RELATED WORK}

The research focus with regards to cliques in the context of complex networks is to come up with efficient heuristics to reduce the run-time complexity in determining the maximum size clique for the entire network graph. Though branch-and-bound has been the common theme among these works, the variation is in the approach used to arrive at the bounds and enforce them in the search space. Strategies used for pruning the search space are typically based on node degree (e.g., [6]), vertex ordering (e.g., [7]) and vertex coloring (e.g., [8]). Recently, a parallelized approach [9] for branch and bound has also been proposed for determining cliques in real-world networks ranging from 1000 to 100 million nodes. Nevertheless, none of the research so far has focused on identifying correlation between the maximal clique size for an individual vertex (the size of the largest clique that a particular vertex is part of) with any of the commonly studied metrics (like node degree, clustering coefficient) for network analysis. Ours is the first step in this direction. With the problem of determining maximum size clique for the entire network graph and maximal size cliques for the individual vertices being NP-hard and computationally time-consuming for complex real-world networks of larger size, it becomes imperative to analyze the correlations of the maximal clique size values of the individual vertices with that of the network metrics that can be easily computed so that meaningful inferences about maximal clique size values can be made.

\section{CliQue}

A clique is a sub graph of a graph in which all the vertices are adjacent to each other. The problems of finding maximum size clique for the entire graph as well as the maximal size cliques for the individual nodes are NP-hard problems [2]. Several exact algorithms (that at the worst case incur exponential time for a NP-hard problem) have been proposed to determine maximum size cliques for sparse graphs. Recently, with the surge in interest to analyze large real-world networks from a graph theoretic point of view, researchers have proposed efficient exact algorithms (e.g., [6-9]) to determine maximum size cliques for large/dense graphs. The common theme [10] behind these algorithms is a branch and bound approach of searching through all possible candidate cliques and limiting the search to only viable candidate sets of vertices whose agglomeration has scope of being a clique of size larger than the currently known clique found as part of the search; the variation among these exact algorithms is the pruning strategy (the approach taken to compute the bounds and use them) to limit the search. In this section, we will describe one such branch and bound-technique based exact algorithm that has been recently proposed in the literature [6] to determine maximum size clique in large network graphs and explain our modification to the algorithm so that it can be used to determine the maximal cliques that each vertex in the graph is part of; the largest among these cliques is the maximum size clique for the entire graph.

Figure 1 outlines the pseudo code of the algorithm (proposed originally in [6]) to determine the maximum size clique for an entire graph. The algorithm starts with an estimate of 0 for the maximum size clique (variable $\max$ ) in the entire graph; the value for max is updated as and when a clique of size larger than the latest value of $\max$ is found. The procedure MAXCLIQUE proceeds in iterations, with each iteration designed to determine the maximum size clique for the entire graph that could also include vertex $v_{i}$ (considered in the increasing order of the IDs). In a particular iteration, vertex $v_{i}$ is considered worthy of exploration for presence in a maximum size 
clique only if its degree is at least the value of $\max$ at that time (i.e., only vertices that could be part of a clique of size larger than the currently known maximum size clique are considered - a pruning strategy). For each such vertex $v_{i}$, a candidate set $U$ of neighbor vertices $v_{j}$ (whose degree is at least the latest value for max) is constructed and passed to the sub routine CLIQUE to find a clique among these vertices; the initial size of the clique is 1 - accounting for $v_{i}$.

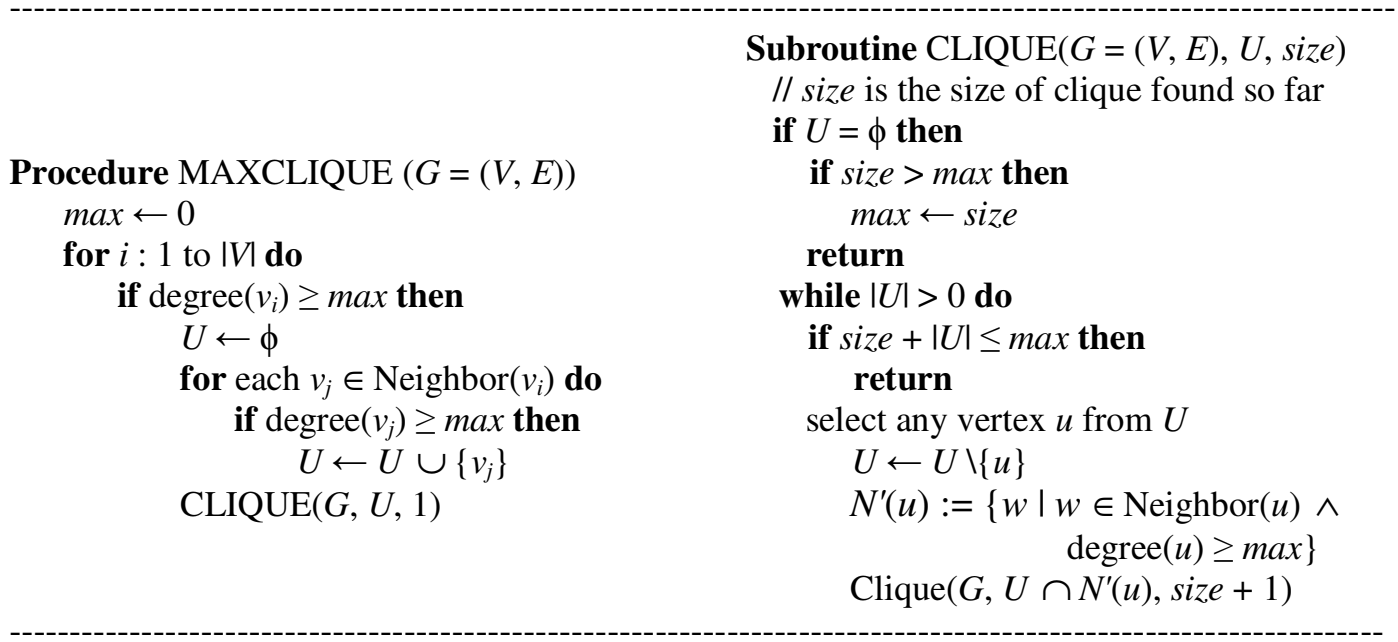

Figure 1. Exact Algorithm to Determine Maximum Size Clique for a Graph (adapted from [6])

The sub routine CLIQUE called with vertex $v_{i}$ as the first constituent vertex of the largest possible clique involving $v_{i}$, expands with one vertex at a time through a combination of iterations and recursions; the sub routine runs as long as the size of the set $U$ is greater than zero or if the current value of $\max$ is less than the sum of the sizes of the set $U$ and the current clique found so far (a pruning strategy). In each such iteration, a vertex $u$ (that is also a neighbor of the starting vertex $v_{i}$ and the other vertices in the clique determined so far) is randomly removed from the set $U$ and the neighbors of $u$ that are also present in $U$ (and hence are neighbors of the starting vertex $v_{i}$ and the other vertices that are part of the clique found so far) are only further considered to be candidates that could be part of the clique, and a recursive call to the CLIQUE sub routine is made with the value of variable size (the size of the largest clique found so far involving vertex $v_{i}$ ) incremented by 1 - accounting for $u$ as the latest entrant in the clique determined so far. Each recursive call to CLIQUE is accompanied by an iteration where a vertex $u$ (that is also a neighbor of the vertices already part of the clique) is removed from the set $U$ passed to the sub routine and only the neighbors of $u$ that are also neighbors of the vertices already in the clique are considered. During any such recursive call, if the size of the set $U$ passed to the sub routine CLIQUE reaches zero, the algorithm terminates the sequence of recursions and updates the value of $\max$ if the size of the clique determined so far involving vertex $v_{i}$ is larger than the current value of $\max$. During the sequence of returns from the recursive calls, it is possible that a new sequence of recursions and iterations is triggered due to the presence of a neighbor $u$ of $v_{i}$ that has scope for being in a clique (involving $v_{i}$ ) of size larger than the clique found so far for the entire graph. The algorithm explores all such possible cliques involving vertex $v_{i}$ that have scope for exceeding the currently known maximum size clique for the entire graph.

At the end, the algorithm returns the maximum size clique for the entire graph that also happens to be the maximal size clique involving some vertex $v_{i}$ such that there is no other vertex $v_{j}(i>j)$ that is also part of the clique. Since the algorithm proceeds with vertices in the increasing order of their IDs, if the maximum size clique for the entire graph involves at least one vertex $v_{i}$ with a smaller ID, the presence of the maximum size clique is detected much earlier and the subsequent 
iterations (with vertices whose IDs are greater than $v_{i}$, but could be part of only cliques of size smaller or equal to the maximum size clique of the entire graph involving $v_{i}$ ) are merely pruned, contributing to the time-efficiency of the algorithm. Hence, the labeling of the vertices with their IDs plays a significant role in the run-time complexity of the algorithm; the algorithm is capable of quickly determining the maximum size clique if the latter comprises of at least one vertex with a smaller ID.

Figure 2 illustrates our modifications (to determine the size of the maximal clique that each vertex is part of) to the pseudo code of the algorithm presented in Figure 1. The tradeoff is an increase in the run-time of the algorithm: we cannot just prune our search based on the vertex IDs; we have to explore the neighborhood of each of the vertices to determine the maximal size clique that each vertex is part of. Since to start with, the maximal size clique known for vertex $v_{i}$ is 0 , there is no need to filter the neighbors of $v_{i}$ in procedure MAXIMALCLIQUE based on the degree of the neighbors; all neighbors of $v_{i}$ are included in the set $U$ and passed onto the sub routine CLIQUE. However, we could retain all of the pruning steps in sub routine CLIQUE (called to find the maximal size clique for each of the vertices $v_{i}$ ) and recursive calls to the same: there is no need to explore the neighbors of vertex $u$ whose degree is less than that of the currently known maximal clique size for vertex $v_{i}$.

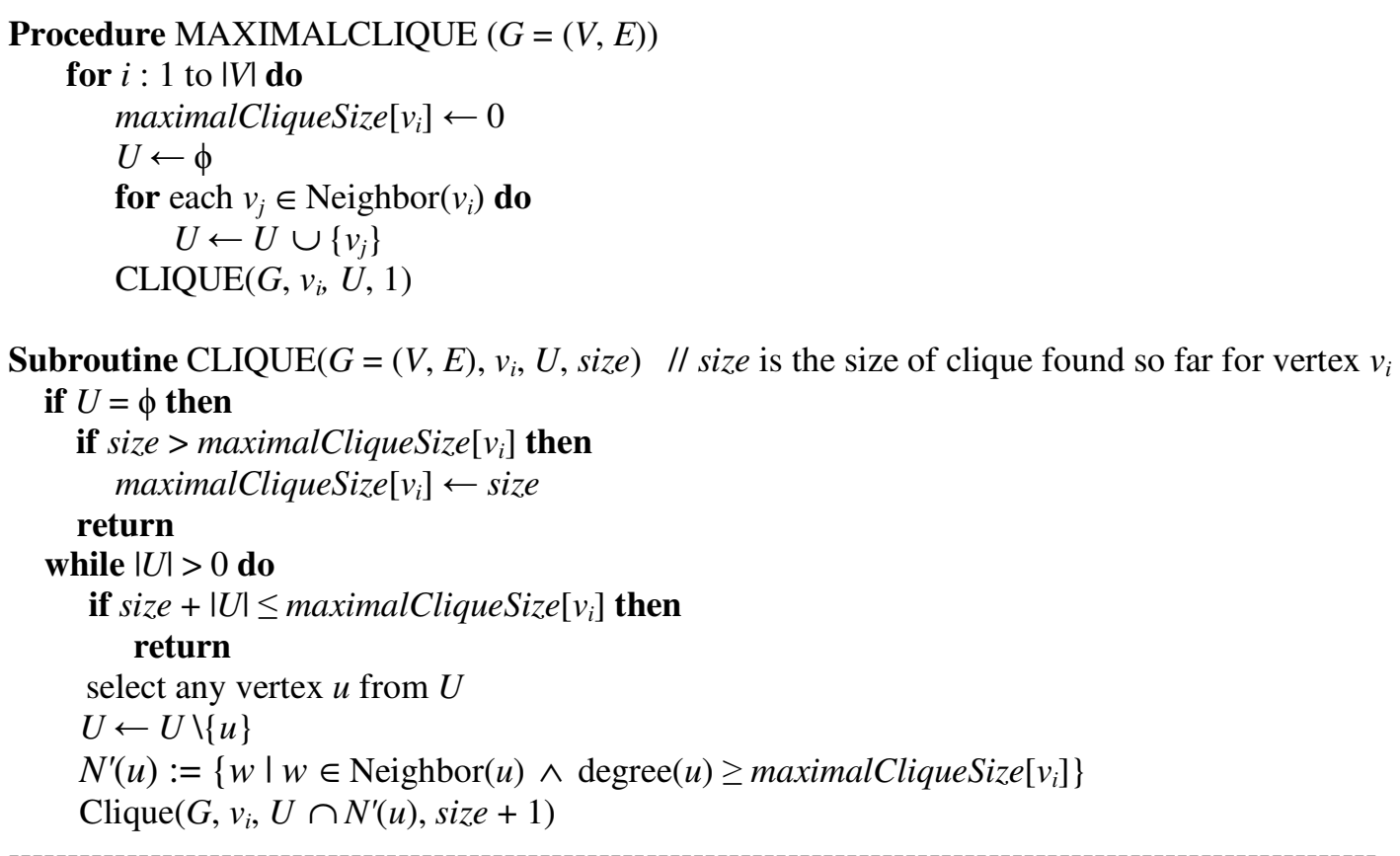

Figure 2.Exact Algorithm to Determine the Maximal Clique Size for each Vertex in a Graph (adapted from [6] )

\section{REAL-WORLD NETWORK GRAPHS AND THEIR ANALYSIS}

In this section, we describe the network graphs analyzed and illustrate the degree distribution and the distribution of the maximal clique size of the vertices in the network graphs. We do so to understand the topological structure of the real-world network graphs as well as to elucidate the impact of the degree and maximal clique size distribution of the vertices on the correlation between the centrality values and the maximal clique size observed for the vertices. The network graphs analyzed are briefly described as follows: (i) Zachary's Karate Club [11]: Social network of friendships (78 edges) between 34 members of a karate club at a US university in the 1970s; 
(ii) Dolphins' Social Network [12]: An undirected social network of frequent associations (159 edges) between 62 dolphins in a community living off Doubtful Sound, New Zealand; (iii) US Politics Books Network [13]: Nodes represent a total of 105 books about US politics sold by the online bookseller Amazon.com. A total of 441 edges represent frequent co-purchasing of books by the same buyers, as indicated by the "customers who bought this book also bought these other books" feature on Amazon; (iv) Word Adjacencies Network [14]: This is a word co-appearance network representing adjacencies of common adjective and noun in the novel "David Copperfield" by Charles Dickens. A total of 112 nodes represent the most commonly occurring adjectives and nouns in the book. A total of 425 edges connect any pair of words that occur in adjacent position in the text of the book; (v) US College Football Network [15]: Network represents the teams that played in the Fall 2000 season of the American Football games and their previous rivalry - nodes (115 nodes) are college teams and there is an edge (613 edges) between two nodes if and only if the corresponding teams have competed against each other earlier; (vi) US Airports 1997 Network: A network of 332 airports in the United States (as of year 1997) wherein the vertices are the airports and two airports are connected with an edge (a total of 2126 edges) if there is at least one direct flight between them in both the directions. Data for networks (i) through (v) can be obtained from http://www-personal.umich.edu/ mejn/netdata/. Data for network (vi) can be obtained from: http://vlado.fmf.uni-lj.si/pub/networks/pajek/data/gphs.htm.
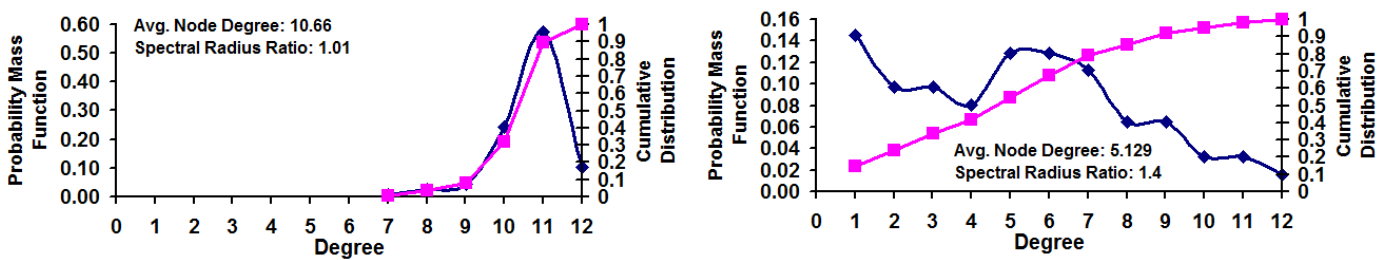

US College Football Network (115 nodes, 613 edges)

Dolphins' Social Network (62 nodes, 159 edges)

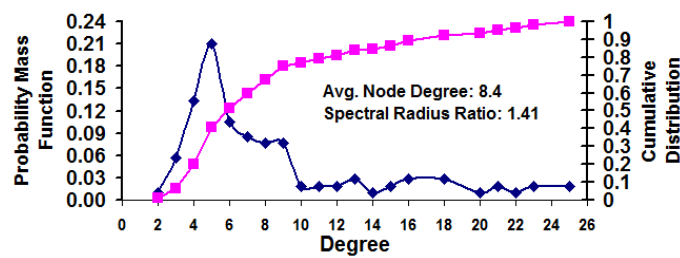

US Politics Books Network (105 nodes, 441 edges)

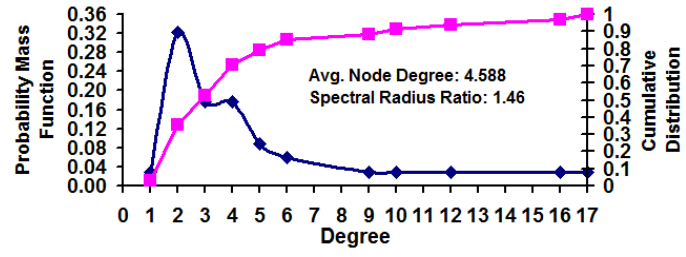

Karate Club Network (34 nodes, 78 edges)

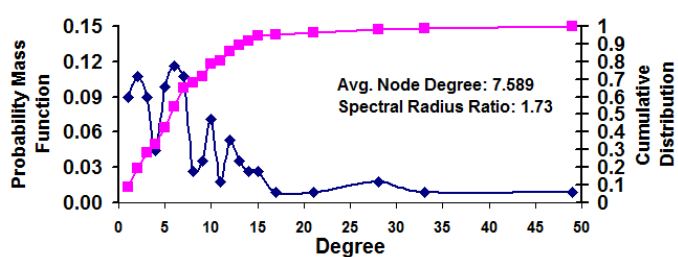

Word Adjacencies Network (112 nodes, 425 edges)

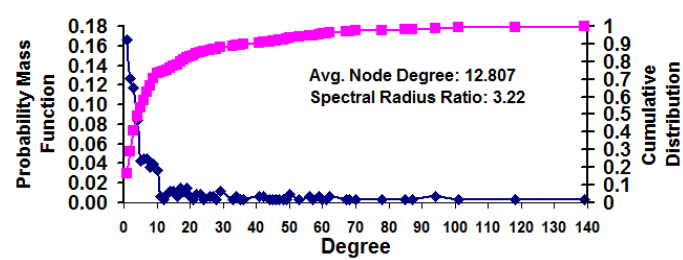

US Airports'97 Network (332 nodes, 2126 edges)

Figure 3. Distribution of Node Degrees (Probability Mass Function and Cumulative Distribution) 


\subsection{Degree Distribution of the Real-World Network Graphs}

Figure 3 presents the degree distribution of the vertices in the six network graphs in the form of both the Probability Mass Function (the fraction of the vertices with a particular degree) and the Cumulative Distribution Function (the sum of the fractions of the vertices with degrees less than or equal to a certain value). We also compute the average node degree and the spectral radius degree ratio (ratio of the spectral radius and the average node degree); the spectral radius (bounded below by the average node degree and bounded above by the maximum node degree) is the largest eigen value of the adjacency matrix of the network graph, obtained as a result of computing the eigenvector centrality of the network graphs. The spectral radius degree ratio is a measure of the variation in the node degree with respect to the average node degree; the closer the ratio is to 1 , the smaller the variations in the node degree and the degrees of the vertices are closer to the average node degree (characteristic of random graph networks). The farther is the ratio from 1, the larger the variations in the node degree (characteristic of scale-free networks). Figure 3 presents the degree distribution of the network graphs in the increasing order of their spectral radius ratio for node degree (1.01 to 3.23). The US College Football network exhibits minimal variations in the degree of its vertices (each team has more or less played against an equal number of other teams). The US Airports network exhibits maximum variation in the degree of its vertices (there are some hub airports from which there are flights to several other airports; whereas there are several airports with only fewer connections to other airports). In between these two extremes of networks, we have the other four network graphs, all of which have a spectral radius ratio for node degree around 1.4-1.7, indicating a moderate variation in the node degree (compared to the spectral radius ratios observed for the US College Football network and the US Airports network).

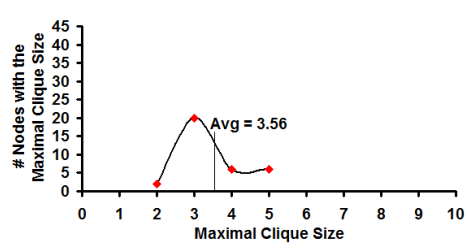

Karate Club Network (34 nodes, 78 edges)

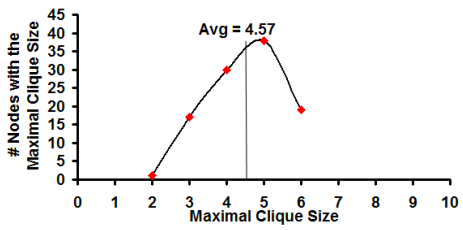

US Politics Books Network (105 nodes, 441 edges)

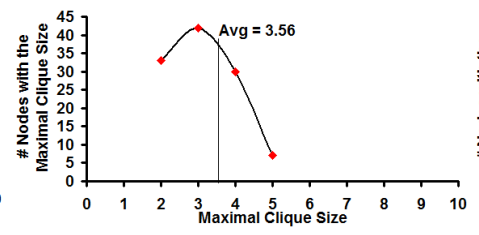

Word Adjacencies Network (112 nodes, 425 edges)

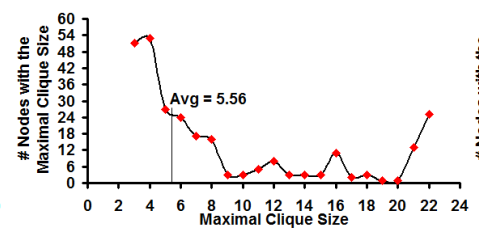

US Airports 1997 Network (332 nodes, 2126 edges)

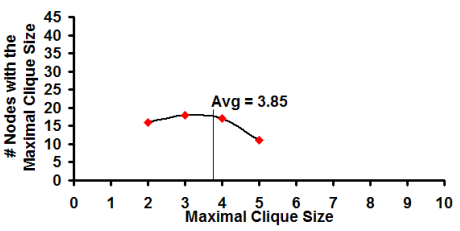

Dolphins' Social Network (62 nodes, 159 edges)

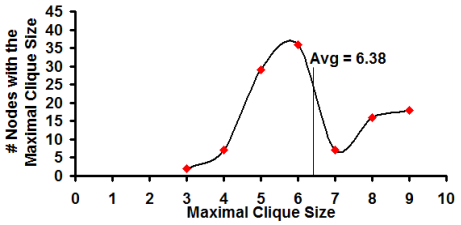

US College Football Network (115 nodes, 613 edges)

Figure 4. Distribution of Maximal Clique Size of the Vertices in Real-World Network Graphs

\subsection{Maximal Clique Size Distribution of the Real-World Network Graphs}

Figure 4 presents the distribution of the maximal clique size of the vertices for the six real-world network graphs, in the increasing order of the average value for the maximal clique size of the vertices. An interesting observation is that five of the six real-world network graphs exhibit a 
Poisson-style distribution for the maximal clique size and the average value of the maximal clique size for the nodes is very close to the maximum value. The only real-world network that does not exhibit a Poisson-style distribution for the maximal clique size is the US Airports network whose distribution of the maximal clique size appears to be more of a scale-free (power-law) pattern with a long tail (wherein the average maximal clique size is 5.56, but there exists a significant number of nodes whose maximal clique size values are 21 and 22). We can also notice that the average value of the maximal clique size of the nodes is not proportional to the number of nodes in the network nor to the spectral radius ratio for node degree. This is evident from three of the six real-world networks with comparable number of nodes (Word Adjacency Network - 112 nodes, US Politics Books Network - 112 nodes and the US Football Network - 105 nodes) incurring significantly different average values for the maximal clique size $(3.56,4.57$ and 6.38 respectively). Similarly, though the spectral radius ratio for node degree increases with increase in the scale-free nature of the networks, we do not observe any such pattern of increase or decrease for the maximal clique size; for example: the US Football Network, Word Adjacency Network and the US Airports Network have spectral radius ratio for node degree values of 1.01, 1.73 and 3.22 respectively; whereas, their average maximal clique size values are $6.38,3.56$ and 5.56 respectively (no pattern of increase or decrease with increase in the spectral radius ratio for node degree).

\section{Correlation Coefficient analysis: MaXimal Clique Size vS. CluStering COEFFICIENT}

The clustering coefficient of a node is the ratio of the number of links between the neighbors of the node to that of the maximum possible number of links between the neighbors of the node [1]. If a node $i$ has $k_{i}$ neighbors and there are $L_{i}$ links among these $k_{i}$ neighbors, then the clustering coefficient for node $i$ is: $C_{i}=\frac{L_{i}}{k_{i}\left(k_{i}-1\right) / 2}$. In this section, we examine whether the clustering coefficient of the nodes in the six real world network graphs is positively correlated to the maximal clique size of the nodes in these graphs. Our reasoning is that a clique comprises of links between any two of its constituent nodes; thus, the neighbors of a node in a clique are also connected with links among themselves. We wanted to examine whether or not this corresponds to links between any two neighbors of a node in the real world network graph itself.

If $\bar{X}$ and $\bar{Y}$ are the average values of the two metrics (say $\mathrm{X}$ and $\mathrm{Y}$ ) observed for the vertices (IDs 1 to $n$, where $n$ is the number of vertices) in the network, the formula used to compute the Correlation Coefficient between two metrics $\mathrm{X}$ and $\mathrm{Y}$ is given in equation (1), as follows:

$$
\operatorname{CorrCoeff}(X, Y)=\frac{\sum_{I D=1}^{n}(X[I D]-\bar{X}) *(Y[I D]-\bar{Y})}{\sqrt{\sum_{I D=1}^{N}(X[I D]-\bar{X})^{2}} \sqrt{\sum_{I D=1}^{N}(Y[I D]-\bar{Y})^{2}}}
$$


Table 1. Correlation Coefficient between Maximal Clique Size and Clustering Coefficient

\begin{tabular}{|l|l|l|l|}
\hline $\begin{array}{l}\text { Network } \\
\text { Index }\end{array}$ & Network Name & $\begin{array}{l}\text { Spectral Radius } \\
\text { Ratio for Node } \\
\text { Degree }\end{array}$ & $\begin{array}{l}\text { Correlation Coefficient: } \\
\text { Maximal Clique Size vs. } \\
\text { Clustering Coefficient }\end{array}$ \\
\hline (vi) & US Airports 1997 Network & 3.22 & -0.47 \\
\hline (i) & Karate Club Network & 1.46 & -0.22 \\
\hline (ii) & Dolphins' Social Network & 1.40 & -0.17 \\
\hline (iv) & Word Adjacencies Network & 1.73 & -0.09 \\
\hline (iii) & US Politics Books Network & 1.41 & 0.07 \\
\hline (v) & US College Football Network & 1.01 & 0.69 \\
\hline
\end{tabular}

Table 1 lists the correlation coefficient observed for the clustering coefficient and the maximal clique size of the nodes for the six real world network graphs (in the order of increasing values of the correlation coefficient), along with the spectral radius ratio for node degree observed for these networks. Contrary to our hypothesis, we observe the clustering coefficient of the nodes in four of the six real world network graphs to be poorly correlated to the maximal clique size of the nodes; the exceptions being the US College Football network (a random network graph) and the US Airports' 97 network (a scale-free network graph) exhibiting respectively moderate levels of positive and negative correlations between the clustering coefficient and the maximal clique size of the nodes. Hence, if at all a positive correlation is observed between the clustering coefficient and maximal clique size, it is most likely by chance. On the other hand, the correlation between the clustering coefficient and maximal clique size turns more negative with increase in the scalefree nature of the networks. For networks that have moderate values of the spectral radius ratio for node degree (that is the networks are neither scale-free nor random), there is pretty much no correlation between the clustering coefficient and maximal clique size of the nodes.

\section{Correlation Coefficient analysis: Maximal Clique Size VS. NODE DEGREE}

In this section, we present the results of correlation coefficient analysis conducted between node degree vis-a-vis the maximal size clique that each vertex is part of. The analysis has been conducted on the six real-world network graphs (mentioned in Section 4) with respect to the node degree and the maximal clique size measured for the vertices in these graphs. We implemented the exact algorithm to determine the maximal clique size for each of the vertices in a graph (see Figure 2). The visualization figures presented in the paper were obtained by porting the network data as well as the node degree/maximal clique size results to Gephi [16] and making appropriate changes to the settings in the latter. The layout algorithm chosen in Gephi for visualization is the Fruchterman Reingold algorithm [17] that presents the network in a circular format (like a globe).

Table 2. Correlation Coefficient between Maximal Clique Size and Node Degree

\begin{tabular}{|l|l|l|l|}
\hline $\begin{array}{l}\text { Network } \\
\text { Index }\end{array}$ & Network Name & $\begin{array}{l}\text { Spectral Radius } \\
\text { Ratio for Node } \\
\text { Degree }\end{array}$ & $\begin{array}{l}\text { Correlation Coefficient: } \\
\text { Maximal Clique Size vs. Node } \\
\text { Degree }\end{array}$ \\
\hline (vi) & US Airports 1997 Network & 3.22 & 0.87 \\
\hline (i) & Karate Club Network & 1.46 & 0.64 \\
\hline (ii) & Dolphins' Social Network & 1.40 & 0.78 \\
\hline (iv) & Word Adjacencies Network & 1.73 & 0.71 \\
\hline (iii) & US Politics Books Network & 1.41 & 0.70 \\
\hline (v) & US College Football Network & 1.01 & 0.32 \\
\hline
\end{tabular}


Table 2 presents a correlation coefficient analysis of node degree and the maximal clique size observed for the vertices in each of the six real-world network graphs (in the decreasing order of the spectral radius ratio for node degree). As we can see in Table 2, in general, the correlation between the node degree and the maximal clique size increases as the spectral radius ratio for node degree increases. This implies, the more scale-free a real-world network is, the higher the correlation between the centrality value and the maximal clique size observed for a node. With several of the real-world networks being mostly scale-free, we expect these networks to exhibit a similar correlation to that observed in this paper. Also, since the correlation between the maximal clique size and node degree is the lowest (correlation coefficient of 0.31 ) for the US College Football Network (a random network), we conjecture that the stronger correlation (correlation coefficient of 0.7 or above) observed between these two metrics in the other five real-world network graphs is not merely by chance.

Figure 5 depicts the correlation observed for the node degree with that of the maximal clique size for the vertices in the real-world network graphs. In these figures, the node size is a measure of the node degree (the larger a node is, the larger is its degree); the node color is a measure of the maximal size clique the vertex is part of (the darker a node is, the larger is the size of the maximal clique for the node). We observe a high correlation between maximal clique size of nodes and nodes with a higher degree as well as located in a neighborhood of high degree nodes. That is, a node with high degree as well as located in a neighborhood of high degree vertices is more likely to be part of a maximal clique of larger size. In addition, as the networks get increasingly scalefree, nodes with high degree are more likely connected to other similar nodes with high degree (to facilitate an average path length that is almost independent of network size: characteristic of scale-free networks [1]) contributing to a positive correlation between degree-based centrality metrics and maximal clique size. We anticipate that as the networks become increasingly scalefree, the hubs (that facilitate shortest-path communication between any two nodes) are more likely to form the maximum clique for the entire network graph - contributing to higher levels of positive correlation between node degree and maximal clique size.

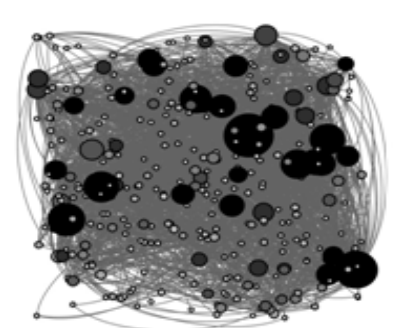

US Airports 1997 Network (332 nodes, 2126 edges)

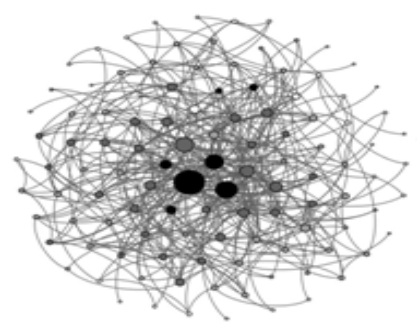

Word Adjacencies Network (112 nodes, 425 edges)

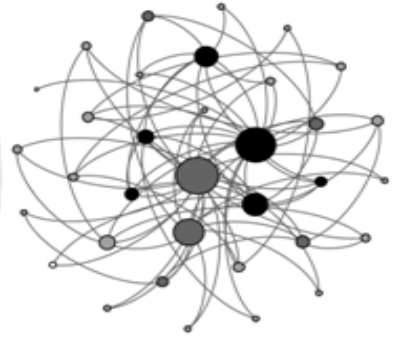

Karate Club Network (34 nodes, 78 edges)

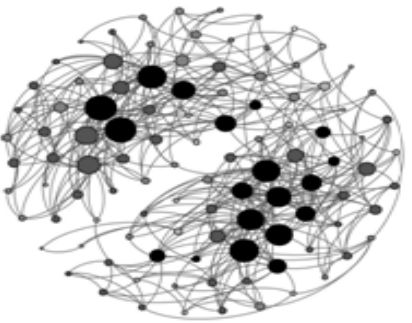

US Politics Books Network (105 nodes, 441 edges)

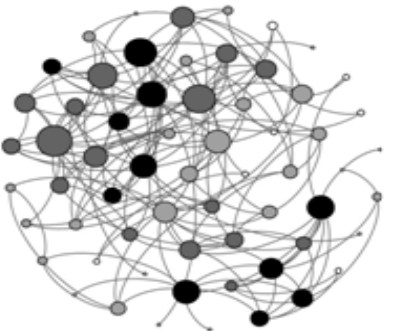

Dolphins' Social Network (62 nodes, 159 edges)

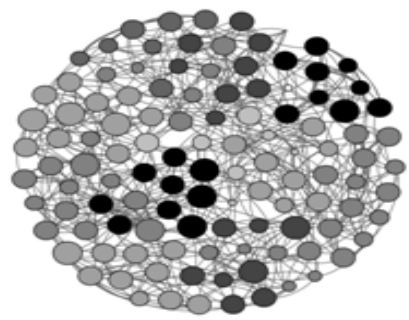

US College Football Network (115 nodes, 613 edges)

Figure 5. Correlation of Maximal Clique Size of the Vertices and Node Degree in the Real-World Network Graphs 


\section{ASSORTATIVITY INDEX-BASED ANALYSIS: MAXIMAL CLIQUE SIZE AND NODE DEGREE}

The assortativity index for a network graph with respect to a particular node-related metric is a measure of the association of nodes with similar values for the metric [1]. For example, the assortativity index of a graph with respect to node degree is a measure of the association of higher degree nodes with other high degree nodes as well as the association of nodes of lower degree nodes with other lower degree nodes. In this section, we analyze the assortativity index of the six real-world network graphs with respect to the maximal clique size and node degree, and examine the nature of association between nodes having higher values for each of these two metrics. If $m$ is the node-related metric of interest, then the assortativity index of the network graph with respect to $m$ is evaluated as the correlation coefficient of the values (with respect to metric $m$ ) for the end nodes of the edges in the graph. Consider a network graph of $n$ nodes and set of undirected (bi-directional) edges $E$; let $m[1], m[2], \ldots ., m[n]$ be the values for nodes $1,2, \ldots, n$ with

respect to metric $m$ and $\bar{m}$ be the average value of the metric, the assortativity index with respect to metric $m$ is given by equation (2).

$$
\operatorname{AssortativityIndex}(m)=\frac{\sum_{(i, j) \in E}(m[i]-\bar{m})^{*}(m[j]-\bar{m})}{\sqrt{\sum_{(i, j) \in E}(m[i]-\bar{m})^{2}} \sqrt{\sum_{(i, j) \in E}(m[j]-\bar{m})^{2}}}
$$

Positive values for the assortativity index with respect to a metric indicates that the network exhibits assortativity with respect to the metric (nodes with higher values for the metric are more likely to be connected to nodes with higher values for the metric and vice-versa); negative values for the assortativity index indicates the network exhibits disassortativity (nodes with lower values for the metric are more likely to be connected to nodes with higher values for the metric and viceversa); assortativity index values close to 0 indicates the network is more neutral with respect to the metric (i.e., the values for the end nodes of the edges with respect to the metric do not exhibit any correlation).

Table 3 lists the assortativity index values for the maximal clique size and degree of the vertices for the six real-world network graphs, along with their spectral radius ratio for node degree. We observe the assortativity index (with respect to the maximal clique size) for all the six network graphs to be positive and the assortativity index value for the maximal clique size increases with increase in the level of randomness in the network, indicating that the association of nodes of a particular maximal clique size with other nodes that are also of the same maximal clique size is more by chance. On the other hand, we observe the assortativity index (with respect to the node degree) for five of the six network graphs (i.e., all network graphs, except the US Football Network that exhibits the characteristic of random graphs) to be negative and the assortativity index values for the node degree gets more negative with increase in the scale-free nature of the network, indicating high degree nodes are more likely to be associated with low degree nodes (especially with increase in the spectral radius ratio for node degree). Finally, to confirm our earlier observation of a positive correlation between maximal clique size of the vertices and node degree, we observe in Table 3 that the six-real world networks could be listed in an identical order, in the increasing order of the Assortativity Index of the network graphs with respect to both maximal clique size of the vertices and node degree. 
Table 3. Assortativity Index of the Real-World Network Graphs based on Maximal Clique Size of the Vertices and Node Degree

\begin{tabular}{|l|l|l|l|l|}
\hline $\begin{array}{l}\text { Network } \\
\text { Index }\end{array}$ & Network Name & $\begin{array}{l}\text { Spectral } \\
\text { Radius Ratio } \\
\text { for Node } \\
\text { Degree }\end{array}$ & $\begin{array}{l}\text { Assortativity } \\
\text { Index for } \\
\text { Maximal } \\
\text { Clique Size }\end{array}$ & $\begin{array}{l}\text { Assortativity } \\
\text { Index for Node } \\
\text { Degree }\end{array}$ \\
\hline (i) & Zachary's Karate Network & 1.46 & 0.13 & -0.48 \\
\hline (vi) & US Airports 1997 Network & 3.22 & 0.17 & -0.21 \\
\hline (iv) & Word Adjacencies Network & 1.73 & 0.20 & -0.09 \\
\hline (iii) & US Politics Books Network & 1.41 & 0.20 & -0.04 \\
\hline (ii) & Dolphins' Social Network & 1.40 & 0.23 & -0.02 \\
\hline (v) & US College Football Network & 1.01 & 0.59 & 0.19 \\
\hline
\end{tabular}

\section{CONCLUSIONS}

The observation of Poisson-style distribution for maximal clique size of the vertices in real-world network graphs irrespective of the number of nodes and the degree distribution of the vertices is an interesting observation that has not been hitherto reported in the literature. We conjecture the distribution for the maximal clique size of the vertices to transform from Poisson to Power-law distribution in networks that are highly scale-free (as observed in the case of the US Airports'97 Network). With the problem of determining maximal clique sizes for individual vertices being computationally time consuming, our approach taken in this paper to study the correlation between maximal clique sizes vis-a-vis node degree and clustering coefficient could be the first step in identifying correlation between cliques/clique size in real-world network graphs and one or more computationally-light node-based network metrics that can be quickly determined and henceforth appropriate inferences can be made about a ranking of the vertices with respect to maximal clique size. The approach taken to first to find the correlation coefficient between the two metrics of interest (like node degree and maximal clique size of the vertices) in the individual network graphs and then ranking the network graphs in the increasing order of the Assortativity Index of the graphs with respect to each of the two metrics (an identical or close to identical listing of the network graphs with respect to the each of the two metrics vindicates the positive correlation observed between the two metrics based on correlation coefficient analysis). Such an approach for correlation study between two node-based metrics is a unique approach that has been so far not presented in the literature. We observe node degree to show promising positive correlations to that of maximal clique sizes of the individual vertices, especially as the networks get increasingly scale-free; this observation could form the basis of future research for analysis of maximal clique size of the vertices in complex real-world network graphs and the correlations of the maximal clique size of the vertices with other computationally-light metrics for complex network analysis.

\section{REFERENCES}

[1] M. Newman, Networks: An Introduction, Oxford University Press, 1st ed., May 2010.

[2] T. H. Cormen, C. E. Leiserson, R. L. Rivest and C. Stein, Introduction to Algorithms, 3rd ed., MIT Press, July 2009.

[3] G. Palla, I. Derenyi, I. Farkas and T. Vicsek, "Uncovering the Overlapping Community Structure of Complex Networks in Nature and Society," Nature, vol. 435, pp. 814-818, 2005.

[4] S. Fortunato, "Community Detection in Graphs," Physics Reports, vol. 486, pp. 75-174, 2010. 
[5] S. Sadi, S. Oguducu and A. S. Uyar, "An Efficient Community Detection Method using Parallel Clique-Finding Ants," Proceedings of IEEE Congress on Evolutionary Computation, pp. 1-7, July 2010 .

[6] B. Pattabiraman, M. A. Patwary, A. H. Gebremedhin, W-K. Liao and A. Choudhary, "Fast Problems for the Maximum Clique Problem on Massive sparse Graphs," Proceedings of the 10th International Workshop on Algorithms and Models for the Web Graph: Lecture Notes in Computer Science, vol. 8305, pp. 156-169, 2013.

[7] R. Carraghan and P. Pardalos, "An Exact Algorithm for the Maximum Clique Problem," Operations Research Letters, vol. 9, no. 6, pp. 375-382, 1990.

[8] P. R. J. Ostergard, "A Fast Algorithm for the Maximum Clique Problem," Discrete Applied Mathematics, vol. 120, no. 1-3, pp. 197-207, 2002.

[9] R. A. Rossi, D. F. Gleich and M. A., Patwary, "Fast Maximum Clique Algorithms for Large Graphs," Proceedings of the 23rd International Conference on World Wide Web Companion, pp. 365-366, April 2014.

[10] E. Tomita and T. Seki, "An Efficient Branch-and-Bound Algorithm for Finding a Maximum Clique," Proceedings of the 4th International Conference on Discrete Mathematics and Theoretical Computer Science, pp. 278-289, 2003.

[11] W. W. Zachary, "An Information Flow Model for Conflict and Fission in Small Groups," Journal of Anthropological Research, vol. 33, no. 4, pp. 452-473, 1977.

[12] D. Lusseau, K. Schneider, O. J. Boisseau, P. Haase, E. Slooten, and S. M. Dawson, "The Bottlenose Dolphin Community of Doubtful Sound Features a Large Proportion of Long-lasting Associations," Behavioral Ecology and Sociobiology, vol. 54, pp. 396-405, 2003.

[13] V. Krebs, http://www.orgnet.com/divided.html, last accessed: March 22, 2015.

[14] M. Newman, "Finding Community Structure in Networks using the Eigenvectors of Matrices," Physics Review, E 74, 036104, September 2006.

[15] M. Girvan and M. Newman, "Community Structure in Social and Biological Networks," Proceedings of the National Academy of Sciences of the United States of America, vol. 19, no. 12, pp. 7821-7826, April 2002.

[16] K. Cherven, Network Graph Analysis and Visualization with Gephi, 1st ed., Packt Publishing, Birmingham, UK, September 2013.

[17] T. M. J. Fruchterman and E. M. Reingold, "Graph Drawing by Force-Directed Placement," Software: Practice and Experience, vol. 21, no. 11, pp. 1129-1164, November 1991. 